The authors performed microTESE in 74 azoospermic men with nonmosaic Klinefelter syndrome, who had not used long-term ( $>1$ year) testosterone replacement therapy preoperatively. Sperm recovery was successful in 42 of 74 men $(56.7 \%)$ overall, and was also successful in 26 of 45 men $(57.7 \%)$ without evident spermatozoa on testicular biopsy. Age was the only factor that influenced the success of microTESE: men in whom sperm recovery was successful were significantly younger than men in whom it was unsuccessful (mean ages 31.6 years versus 35.0 years, $P=0.002$ ). The threshold age of 30.5 years had the best sensitivity and specificity $(78 \%$ and $48 \%)$ in predicting the success of microTESE. There was an inverse correlation between age and the success of microTESE.

The authors conclude that men with nonmosaic Klinefelter syndrome who undergo microTESE before 30 years of age have a better chance of successful sperm recovery than older men have. They suggest microTESE success rates might be highest in young men, but warn that the effect of long-term testosterone-replacement therapy on the success of microTESE has not been adequately studied.

Original article Bakircioglu ME et al. (2006) Aging may adversely affect testicular sperm recovery in patients with Klinefelter syndrome. Urology 68: 1082-1086

\section{Active treatment reduces mortality in men with low to intermediate risk prostate cancer}

The proportion of men with a diagnosis of earlystage, low to intermediate grade, localized prostate cancer has increased as a result of PSA screening, although whether these patients should be managed with active treatment or watchful waiting is a subject of much debate. Wong et al. have conducted an observational cohort study in the US. Their results suggest that there is a significant survival advantage for patients $>65$ years of age who undergo active treatment over patients who undergo watchful waiting.

Data for 44,630 men (aged 65-80 years) with localized, well or moderately differentiated, stage T1-T2, Gleason score 2-7 prostate cancer and $>1$ year survival after diagnosis were obtained from Medicare records and the Surveillance, Epidemiology, and End Results (SEER) database. These patients were grouped according to whether they had received ('treatment group', $n=32,022)$ or had not received ('observation group', $n=12,608$ ) active treatment (radical prostatectomy or radiation therapy) within 6 months of diagnosis.

Patients in the treatment group were $30 \%$ less likely to die during the 12-year follow-up period than those in the observation group. This association between active treatment and mortality was consistent after adjustment for a considerable number of confounding variables, such as comorbidity, sociodemographic background, ethnicity, age, and tumor variables.

The results of this observational study require validation by randomized, controlled trials; although selection bias might have influenced the outcome of the study, the survival advantage associated with treatment was consistent across the healthiest and sickest patients.

Original article Wong Y-N et al. (2006) Survival associated with treatment vs observation of localized prostate cancer in elderly men. JAMA 296: 2683-2693

\section{Familial association between prostate and breast cancer in African-American siblings}

African-American men are $60 \%$ more likely than white men to receive a prostate cancer diagnosis. In the general population, prostate-cancer risk is increased in patients with a family history of prostate or breast cancer, particularly in siblings. Beebe-Dimmer and colleagues have now reported their findings from a population-based, case-control study of this association among African-American participants in the Flint Men's Health Study.

Detailed family histories were obtained from 121 men with prostate cancer (mean age at diagnosis 65 years) and 179 men without prostate cancer (mean age at survey 61 years).

Men with prostate cancer were more likely than controls to have at least one brother with prostate cancer (age-adjusted odds ratio 4.80; $95 \% \mathrm{Cl} 2.01-11.44)$ or a sister with breast cancer (age-adjusted odds ratio $3.80 ; 95 \% \mathrm{Cl}$ 1.57-9.22). Age at diagnosis of affected brothers was similar in cases and controls (63.6 years versus 62.3 years respectively). Sisters of men with prostate cancer tended to be younger at breast-cancer diagnosis than sisters of control men (49.3 years versus 61.9 years respectively), although the difference was not statistically significant, probably because few affected sisters 